\title{
Uma Abordagem de Regressão Múltipla para Validação de Variáveis de Autorregulação da Aprendizagem em Ambientes de LMS
}

Rodrigo Lins Rodrigues ${ }^{1}$, João C. Sedraz Silva², Jorge L. C. Ramos², Fernando d. F. de Souza $^{3}$, Alex Sandro Gomes ${ }^{3}$.

\author{
${ }^{1}$ Universidade Federal Rural de Pernambuco \\ Recife-PE, Brasil. \\ ${ }^{2}$ Universidade Federal do Vale do São Francisco \\ Juazeiro - BA - Brasil \\ ${ }^{3}$ Universidade Federal de Pernambuco \\ Recife-PE, Brasil \\ rodrigo.linsrodrigues@ufrpe.br \\ \{joao.sedraz,jorge.cavalcanti\}@univasf.edu.br \\ $\{$ asg, fdfd\}@cin.ufpe.br
}

\begin{abstract}
This study aims to investigate the applicability of the multiple regression model for the validation of behavioral learning self-regulation variables. a survey of 408 participants courses in distance education mode with an average age of 30 was conducted. Data collection was done through a hybrid approach between questionnaire and log files of variables, in order to examine whether the statement of the students, answered in the questionnaire was explained by its behavior within the LMS platform. As a result, it was validated a set of thirteen significant variables for the learning of selfregulation of behavior of students in Moodle.
\end{abstract}

Resumo. O presente estudo visa investigar a aplicabilidade do modelo de Regressão Múltipla para a validação de variáveis comportamentais de autorregulação da aprendizagem em contexto de EAD. Foi realizada uma pesquisa com 408 participantes de cursos na modalidade EAD com idade média de 30 anos. A coleta dos dados foi feita através de uma abordagem híbrida entre questionário e variáveis de arquivos de log, com o objetivo de analisar se a afirmação dos alunos, respondida através do questionário, era explicada pelo seu comportamento dentro da plataforma de LMS. Como resultado, foi validado um conjunto de treze variáveis significativas referentes ao comportamento de autorregulação da aprendizagem dos alunos na plataforma Moodle.

\section{Introdução}

Estudos vêm sendo desenvolvidos com o intuito de analisar a importância de habilidades da autorregulação para o aprendizado em ambientes de EAD (Barnard, et al. 2008); (Ali, et al. 2014). A autorregulação da aprendizagem é a habilidade cognitiva por meio da qual as pessoas conseguem gerir recursos, o tempo, os esforços, o ambiente, a interação e a busca por ajuda de no processo de aprendizagem (Chen 2002); (Pintrich 1999); (Zimmerman e Martinez-Pons 1988).

Estudantes que detém destas habilidades conseguem controlar o ritmo, o direcionamento do processo de aprendizagem e gerenciar um conjunto de características do ambiente de aprendizagem, realizando escolhas que o auxiliam no desenvolvimento de suas funções cognitivas (Broadbent e Poon 2015) . 
V Congresso Brasileiro de Informática na Educação (CBIE 2016)

Anais do XXVII Simpósio Brasileiro de Informática na Educação (SBIE 2016)

Pesquisadores acreditam que os ambientes de aprendizagem on-line exigem do aluno uma maior consciência de seu processo de aprendizagem autorregulado (Dabbagh e Kitsantas 2005); (Schunk e Zimmerman 1998). Estes autores acreditam que as habilidades de autorregulação são essenciais para o sucesso da aprendizagem autônomas (autodirigidas) e que o desenvolvimento dessas habilidades podem ser suportados por ferramentas pedagógicas baseadas na Web (Azevedo 2005); (Dabbagh e Kitsantas 2004).

No entanto, ainda existem poucas pesquisas que objetivam a mensuração de características comportamentais de autorregulação em ambientes virtuais de aprendizagem e cursos na modalidade de EAD. A maioria dos instrumentos de coleta utilizados na literatura foram concebidos para serem aplicados no ensino presencial.

Diante deste contexto este artigo tem como objetivo a identificação e validação de variáveis, em ambientes de LMS, que possibilitem identificar traços comportamentais de autorregulação da aprendizagem de alunos em cursos na modalidade de EAD, possibilitando assim que se possa aplicar técnicas de estatística e mineração de dados para tomadas de decisões em tempo real.

\section{Abordagens de mensuração de SRL na literatura}

Quantificar habilidades de autorregulação do aluno, durante as tarefas específicas de aprendizagem é um desafio. A maioria das abordagens de mensuração são realizadas através de coleta com questionários.

\subsection{Questionários como instrumentos de coleta}

Um dos instrumentos utilizados na literatura é o questionário MSLQ (Yukselturk e Bulut 2009), (Adesope, Zhou e Nesbit 2015), (Panadero, et al. 2015), (Sanchez-Santillan, et al. 2016), (Ali, et al. 2014). Este instrumento é um dos mais utilizado na literatura, embora uma de suas limitações seja o fato do mesmo ter sido construído para ser utilizado em ensino presencial, não levando em considerações aspectos peculiares da modalidade de EAD.

Outro instrumento utilizado na literatura foi desenvolvido por (Barnard, Lan, et al. 2009) intitulado OSLQ. Este instrumento foi desenvolvido e projetado para a avaliação de habilidades de autorregulação que são importantes na aprendizagem online. Suas subescalas incluem estruturação ambiente, estabelecimento de metas, gestão do tempo, a procura de atendimento, estratégias de tarefas de auto-avaliação.

Embora os instrumentos de coleta através de questionários, sejam os mais utilizados pela literatura, existem várias limitações no que diz respeito a: necessita de vários dias para a coleta de dados, são baseadas na opinião do aluno sobre seu próprio comportamento e não levam em consideração as reais ações comportamentais do aluno no seu processo de ensino e aprendizagem.

Alguns autores questionam a validade das abordagens de mensuração através de questionários, estas abordagens caracterizam-se pela auto afirmação retrospectiva do aluno sobre suas ações de aprendizagem. Autores como (Winne e Perry 2000) e (Winne e Jamieson-Noel 2002), tem duvidado da eficácia destas abordagens, pois afirmam que em alguns casos os alunos podem não serem capazes de lembrar com precisão suas 
V Congresso Brasileiro de Informática na Educação (CBIE 2016)

Anais do XXVII Simpósio Brasileiro de Informática na Educação (SBIE 2016)

atividades no passado, podem subestimar seu próprio comportamento dando respostas tendenciosas e em casos mais extremos podem não responder tais perguntas com honestidade.

\subsection{Arquivos de log como instrumentos de coleta}

Uma segunda abordagem de coleta de dados é através de arquivos de log. Os principais trabalhos que utilizam essa abordagem são os trabalhos de (Schoor e Bannert 2012), (Sabourin, Mott e Lester 2012), (Bondareva, et al. 2013), (Nussbaumer, et al. 2015), (Segedy, Kinnebrew e Biswas 2015), (Sonnenberg e Bannert 2015), (You 2016). A maioria destes trabalhos utilizam dados de softwares educacionais específicos para o e-Learning e não focam em dados de plataformas de LMS.

Dentro da perspectiva de mensuração através de arquivos de $\log$ em plataformas de LMS, um dos trabalhos que merece destaque é o trabalho do (You 2016). Neste estudo o autor identifica indicadores comportamentais para a aprendizagem autorregulada em sistemas de LMS tentando prever o sucesso dos alunos ao termino de um curso. Neste trabalho o autor utilizou regressão linear para construção de modelos e verificação de variáveis significativas. Uma das limitações deste trabalho é a pouca profundidade teórica na escolha das variáveis explicativas.

Diante desta problemática, as próximas seções abordam o método desta pesquisa que busca identificar e validar as principais variáveis comportamentais em ambientes de LMS no que diz respeito aos construtos de autorregulação da aprendizagem. Estas variáveis possibilitarão que pesquisadores que tenham interesse neste fenômeno, possam realizar inferências em tempo real utilizando um conjunto de variáveis representativas do fenômeno.

\section{Método}

Neste trabalho foi utilizado a técnica estatística de Análise de Regressão Linear Múltipla com dados de questionário e dados comportamentais extraídos de arquivos log.

Inicialmente foi aplicado o questionário de autorregulação da aprendizagem OSLQ (Barnard, Lan e To, et al. 2009). Em seguida foram identificadas e extraídas as variáveis comportamentais do banco de dados da plataforma Moodle através de logs, que representam os comportamentos dos alunos de acordo com os construtos definidos pela teoria de autorregulação da aprendizagem.

De posse de ambas as bases de dados, foi realizado uma integração, através da chave primária de ambas as bases de dados. A função desta fase de integração foi construir uma só base que contivesse dados do questionário e dados comportamentais, com o objetivo de analisar se a afirmação dos alunos, respondida através do questionário, era explicada pelo seu comportamento dentro da plataforma de LMS.

\subsection{Público alvo e Amostra}

Para a aplicação do instrumento, foi utilizada uma amostra coletada através da Universidade de Pernambuco que promove, a mais de dez anos, cursos de graduação na modalidade de EAD através do programa Universidade Aberta do Brasil. 
V Congresso Brasileiro de Informática na Educação (CBIE 2016)

Anais do XXVII Simpósio Brasileiro de Informática na Educação (SBIE 2016)

Os dados foram coletados através de um processo de amostragem aleatória simples (Bolfarine e Bussab 2005), totalizando uma amostra de 408 alunos, com idade média de $30(\mathrm{SD}=18,23)$ anos coletada durante um período de dois meses. A Tabela 1 mostra a frequência do quantitativo de alunos por curso.

Tabela 1. Variáveis de interação coletadas.

\begin{tabular}{l|cccc}
\hline Cursos & Frequência & Freq. Rel. & Freq. Perc. & Freq. Acum. \\
Administração Pública & 27 & 0,07 & 6,62 & 6,62 \\
Licenciatura em Biologia & 112 & 0,27 & 27,45 & 34,07 \\
Licenciatura em Letras & 88 & 0,22 & 21,57 & 55,64 \\
Licenciatura em Pedagogia & 170 & 0,42 & 41,67 & 97,30 \\
Não Informado & 11 & 0,00 & 2,70 & 100,00 \\
$\quad$ Total & $\mathbf{4 0 8}$ & & & \\
\hline
\end{tabular}

Dos alunos que fizeram parte da amostra $57,7 \%$ possuem apenas ensino médio, $24,22 \%$ já cursaram algum curso superior, seja completo ou parcialmente e 7,35\% possuem pós-graduação, nos níveis especialização, mestrado ou doutorado.

\subsection{Instrumentos de coleta Questionário OSLQ}

O instrumento de coleta utilizado nesta pesquisa foi adaptado do questionário de autorregulação da aprendizagem on-line (OSLQ) desenvolvido por (Barnard, Lan e Crooks, et al. 2008) e (Barnard, Lan, et al. 2009). Este instrumento é uma escala de 24 itens com um formato de resposta do tipo escala de Likert de 5 pontos com valores variando de 'concordo totalmente' (5) até 'discordo totalmente' (1).

Como as competências de autorregulação da aprendizagem parecem ser "altamente dependentes de contexto" (Schunk 2005), o desenvolvimento de um instrumento adaptado para os ambientes de aprendizagem on-line é uma etapa necessária em pesquisas que avaliam o fenômeno na modalidade de ensino à distância. Nas pesquisas de (Barnard, Lan, et al. 2009) a autora avaliou a manifestação das competências de autorregular a aprendizagem em ambientes de aprendizagem online com uma amostra de 434 estudantes de Universidades localizadas no sudoeste dos Estados Unidos. Para este estudo realizamos, após a aplicação do questionário, uma avaliação do mesmo, através da análise de adequação do instrumento utilizando os valores de Alpha de Cronbach.

\subsection{Variáveis comportamentais de SRL}

O questionário, descrito na seção anterior, serviu para que pudéssemos fazer um mapeamento de variáveis comportamentais na plataforma LMS Moodle, afim de obter variáveis que representassem os itens do questionário, não apenas de forma opinativa, mas como uma comprovação da opinião do aluno, através da extração do seu próprio comportamento nos registros de log da plataforma Moodle. Para tal, as variáveis foram mapeadas e agrupadas dentro dos construtos de autorregulação da aprendizagem, como mostrado na Tabela 1.

Tabela 1. Variáveis mapeadas do LMS Moodle.

\begin{tabular}{|c|l|c|}
\hline Variável & Descrição sobre as variáveis & \multirow{2}{*}{ Construto } \\
\hline VAR01 & Quantidade de diferentes locais (IP's) a partir dos quais a(o) aluna(o) acessou o ambiente. & Estrut. do ambiente \\
\hline VAR02 & Quantidade de mensagens enviadas por aluna(o) às(os) Professoras(es) pelo ambiente. & \multirow{2}{*}{} \\
\hline VAR03 & Quantidade de mensagens enviadas por aluna(o) às(os) Tutor(es) pelo ambiente. & \\
\cline { 1 - 2 } VAR04 & Quantidade geral de mensagens enviadas pela(o) aluna(o) dentro do ambiente. &
\end{tabular}


V Congresso Brasileiro de Informática na Educação (CBIE 2016)

Anais do XXVII Simpósio Brasileiro de Informática na Educação (SBIE 2016)

\begin{tabular}{|c|c|c|}
\hline VAR05 & Quantidade geral de mensagens recebidas pela(o) aluna(o) dentro do ambiente. & \multirow{6}{*}{ Busca por Ajuda } \\
\hline VAR06 & Quantidade de tópicos criados pelo(a) aluno(a) em fórum do tipo "tira-dúvidas". & \\
\hline VAR07 & Quantidade de postagens no "Fórum tira dúvidas"; & \\
\hline VAR08 & Quant. de postagens de um(a) aluno(a) em fóruns que foram respondidas por outros(as) & \\
\hline VAR09 & Quantidade de postagens de um(a) aluno(a) em fóruns que foram respondidas pelo(a) & \\
\hline VAR10 & Quantidade de colegas diferentes para quem o(a) aluno(a) enviou mensagens dentro do & \\
\hline VAR12 & Quantidade de visualizações da aba "Conteúdo" do curso, onde constam os arquivos com o & \multirow{8}{*}{$\begin{array}{c}\text { Estratégias para } \\
\text { Realização de Tarefas }\end{array}$} \\
\hline VAR13 & Horário que mais realizou atividades; & \\
\hline VAR14 & Turno do dia em que realizou mais atividades. & \\
\hline VAR15 & Quantidade de atividades entregues por um(a) aluno(a) no prazo, por disciplina; & \\
\hline VAR16 & Quantidade de atividades entregues por um(a) aluno(a) fora do prazo, por disciplina; & \\
\hline VAR17 & Tempo médio entre a abertura da atividade e sua submissão; & \\
\hline VAR18 & Quantidade de leituras feitas ao fórum (pageviews); & \\
\hline VAR19 & Quantidade de postagem feitas ao fórum; & \\
\hline VAR20 & Quantidade de respostas ao tópico principal (refazer opinião em fórum); & \multirow{4}{*}{ Autoavaliação } \\
\hline VAR21 & Quantidade de pageviews ao quadro de notas; & \\
\hline VAR22 & Quantidade de vezes que o aluno visualiza o (Cheklist Atividades) & \\
\hline VAR23 & Quantidade de visualizações de notas por atividade; & \\
\hline VAR24 & Média semanal da quantidade de acessos de um(a) aluno(a) ao ambiente. & \multirow{12}{*}{$\begin{array}{l}\text { Gerenciamento do } \\
\text { tempo }\end{array}$} \\
\hline VAR25 & Tempo médio entre a criação de um tópico no fórum temático e a primeira postagem do & \\
\hline VAR28 & Quantidades de Time Out; & \\
\hline VAR29 & Tempo médio de realização de uma atividade; & \\
\hline VAR30 & Tempo médio entre a abertura da atividade e sua realização; & \\
\hline VAR31 & Quantidade de acessos do(a) aluno(a) ao ambiente. & \\
\hline VAR31b & Quantidade de dias distintos que o aluno entrou na disciplina & \\
\hline VAR31c & Quantidade de dias distintos que o aluno entrou na plataforma & \\
\hline VAR32a & Quantidade de acessos do(a) aluno(a) ao ambiente por turno (Manhã). & \\
\hline VAR32b & Quantidade de acessos do(a) aluno(a) ao ambiente por turno (Tarde). & \\
\hline VAR32c & Quantidade de acessos do(a) aluno(a) ao ambiente por turno (Noite). & \\
\hline VAR32d & Quantidade de acessos do(a) aluno(a) ao ambiente por turno (Madrugada). & \\
\hline VAR33 & Quantidade de atividades entregues por um(a) aluno(a) no prazo, por disciplina; & \multirow{3}{*}{$\begin{array}{l}\text { Estabelecimento de } \\
\text { metas }\end{array}$} \\
\hline VAR34 & Quantidade geral de postagens de um(a) aluno(a) em fóruns. & \\
\hline VAR35 & Quantidade de respostas de um(a) professor(a) para as dúvidas de alunos(as) em fóruns. & \\
\hline
\end{tabular}

Foram elaborados trinta e cinco scripts $S Q L$ para a extração das variáveis na base de dados do Moodle. Todas as variáveis comportamentais extraídas, foram referente a comportamentos dos alunos durante o período de um ano. Em seguida foi realizada a junção dos dados coletados com o questionário e os dados comportamentais coletados através da base de dados do Moodle.

\section{Análise e Interpretação dos dados}

Para as análises deste artigo foi utilizado o software estatístico $\mathrm{R}^{1}$. Para a validação do questionário, através cálculo dos valores de alpha de Cronbach, foi utilizado o pacote $p s y^{2}$ onde posteriormente foi realizado a análise de Regressão Linear Múltipla.

\subsection{Validação do questionário}

O questionário é composto por 24 itens, onde é subdividido um quantitativo de itens que representa cada um dos seis construtos. Para a validação do questionário foi utilizado os valores de Alpha de Cronbach com o intuito de verificar o índice de consistência interna dos construtos do questionário. Este índice foi inicialmente

\footnotetext{
${ }^{1}$ https://www.r-project.org/

${ }^{2}$ https://cran.r-project.org/web/packages/psy/psy.pdf
} 
V Congresso Brasileiro de Informática na Educação (CBIE 2016)

Anais do XXVII Simpósio Brasileiro de Informática na Educação (SBIE 2016)

apresentado por Lee J. Cronbach (1951), como uma forma de verificar a confiabilidade de um instrumento de coleta tipo questionário.

Os resultados para os valores de alpha de Cronbach para cada construto foram: 'estabelecimento de metas' $(\alpha=0,59)$, 'estruturação do ambiente' $(\alpha=0,79)$, 'estratégia para realização de tarefas' $(\alpha=0,74)$, 'gerenciamento do tempo' $(\alpha=0,75)$, 'busca por ajuda' $(\alpha=0,74)$ e 'autoavaliação' $(\alpha=0,72)$.

É importante mencionar que não existe um consenso na literatura acerca da interpretação da confiabilidade de um questionário obtida a partir do valor deste coeficiente. Não há um valor mínimo definido para o coeficiente alfa de Cronbach ser aceito como bom, mas se acha na literatura o valor de 0,70 como aceitável (Freitas e Rodrigues 2005); (Urdan 2001); (Oviedo e Campo-Arias 2005); (Milan e Trez 2005). Embora existam autores que utilizam o valor de alfa sem fazerem nenhuma menção a um mínimo aceitável (Salomi, Miguel e Abackerli 2005); (Miguel e Salomi 2004); (Mato e Veiga 2000).

Nesta validação apenas o construto Estabelecimento de metas obteve valor de coeficiente Alpha de Cbronbach abaixo de 0,07. Estes resultados foram importantes para darmos prosseguimento as análises com a aplicação da análise de regressão múltipla.

\subsection{Validação das variáveis comportamentais}

Para a validação das variáveis comportamentais foi aplicado o modelo de Regressão Múltipla, onde existe o interesse em avaliar a relação de uma variável $Y$ (variável dependente ou variável resposta) em relação a $k$ variáveis $X j$ (variáveis independentes ou covariáveis) $j=1,2, \ldots, k$ O objetivo foi reduzir um grande número de variáveis para poucas dimensões com o mínimo de perda de informação, permitindo a detecção dos principais padrões de similaridade, associação e correlação entre as variáveis de acordo com os construtos (Cameron, e Trivedi 1998). Tal modelo é apresentado na seguinte equação:

$$
Y_{i}=\beta_{0}+\beta_{i 1} X_{1}+\beta_{2} X_{i 2}+\cdots+\beta_{k} X_{n k}+\mathcal{E}_{i}, \quad i=1, \ldots, n
$$

em que, $n$ é o número de indivíduos, $y i$ é a observação da variável dependente para o $i$ ésimo indivíduo, $x_{i}=\left(x_{i 1}, x_{i 2}, \ldots, x_{i k}\right)^{\prime}$ é um vetor de observações das variáveis independentes para o $i$-ésimo indivíduo, $\beta=\left(\beta_{0}, \beta_{1}, \beta_{2} \ldots, \beta_{k}\right)^{\prime}$ é um vetor de coeficientes de regressão (parâmetros) e $\varepsilon_{i}$ é um componente de erro aleatório. Assumese que esses erros são independentes e seguem distribuição normal com média zero e variância desconhecida $\sigma^{2}$.

Neste artigo a variável resposta $(Y i)$ é tida como a média dos itens do questionário que representa cada construto de autorregulação da aprendizagem e as variáveis independentes $X_{n}$ são as variáveis que representam os comportamentos de autorregulação dos alunos, extraídos da base de dados da plataforma Moodle.

Para a análise foi realizado a construção de seis modelos desenvolvidos referentes aos seguintes construtos: (1) Estabelecimento de Metas, (2) Estruturação do Ambiente (EA), (3) Estratégias para realização de Tarefas (ET), (4) Gerenciamento do Tempo (GT), (5) Busca Por Ajuda (BA) e (6) Auto Avaliação (AA). 
V Congresso Brasileiro de Informática na Educação (CBIE 2016)

Anais do XXVII Simpósio Brasileiro de Informática na Educação (SBIE 2016)

Através do modelo de Regressão Múltipla, para cada um dos construtos, foi obtido o valor de significância para cada uma das variáveis. O objetivo foi identificar quais variáveis comportamentais melhor explicam o construto identificado através do questionário, ou seja, identificar os principais comportamentos de autorregulação dos alunos que explicassem suas afirmações através do questionário OSQL (Barnard, Lan e To, et al. 2009).

A tabela 2 mostra a variáveis mais significativas, que melhor explicam o construto.

Tabela 2. Significância das variáveis para cada um dos construtos

\begin{tabular}{l|l|l}
\hline Construto & Variável & Significância(p-valor) \\
\hline Estruturação do Ambiente (EA) & VAR01 & 0,0366 \\
\hline Busca Por Ajuda (BA) & VAR02, VAR03, VAR04, & 0,$0304 ; 0,0309 ; 0,0433 ; 0,0472 ;$ \\
\hline Estratégias para realização de Tarefas (ET) & VAR18 & 0,0438 \\
\hline Auto Avaliação (AA) & VAR22 & 0,000435 \\
\hline Gerenciamento do Tempo (GT) & VAR28, VAR29, VAR31b, & 0,$044961 ; 0,000612 ; 0,004469 ;$ \\
\hline Estabelecimento de Metas (EM) & VAR33, VAR34 & $4,91 \mathrm{e}-07 ; 0,0013$ \\
\hline *Variáveis com p-valor acima de 0,05 não possui significância estatística
\end{tabular}

O primeiro modelo especificado foi o modelo de Estruturação do ambiente $(E A)$. Este construto, apesar de existir no questionário, foi difícil apontar uma variável comportamental dentro do ambiente que pudesse representar a estruturação do seu próprio ambiente de trabalho do aluno. Neste caso apenas a variável (VAR01) foi extraída, que trata da quantidade de diferentes locais $\left(I P^{\prime} s\right)$ a partir dos quais a(o) aluna(o) acessou o ambiente. Esta variável nos demonstra o quanto o aluno, durante um semestre, permanece estudando em um só local de trabalho ou em locais e dispositivos diversificados. $\mathrm{O}$ resultado deste modelo apontou uma significância estatística para esta variável ( $p$-valor 0,0366).

O segundo modelo foi o que obteve o maior número de variáveis significativas, este modelo teve como variável dependente o construto Busca por Ajuda (BA). Dentre todas as variáveis incluídas no modelo, cinco delas tiveram significância estatística (VAR02, VAR03, VAR04, VAR05, VAR10). Todas as variáveis significativas se referem a troca de mensagens privadas entre aluno e professor, aluno e tutor em busca de ajuda.

O terceiro modelo desenvolvido foi referente as Estratégias para Realização de Tarefas (ET). Este modelo foi construído por oito variáveis comportamentais, que inicialmente representavam o comportamento do aluno em traços dentro da plataforma que indicavam estratégias para realização de tarefas. Após a aplicação do modelo de Regressão de Múltipla, foi verificado que apenas a variável (VAR18) que trata da quantidade de leituras feitas ao fórum (pageviews) teve significância estatística para representar o construto de estratégias para realização de tarefas.

O quarto modelo foi relacionado ao construto Auto Avaliação (AA). Inicialmente para este construto foram apontadas quatro variáveis comportamentais, no entanto apenas a variável (VAR22), que trata da quantidade de vezes que o aluno visualiza o checklist de atividades realizadas, essa funcionalidade permite o aluno consultar seu status de realização de atividades dentro da plataforma.

O quinto construto, referente ao Gerenciamento do Tempo $(G T)$ foi o segundo modelo que teve mais variáveis significativas (VAR28, VAR29, VAR31b,VAR31c). A 
V Congresso Brasileiro de Informática na Educação (CBIE 2016)

Anais do XXVII Simpósio Brasileiro de Informática na Educação (SBIE 2016)

variável 28 e 29 são relacionadas ao tempo que o aluno gasta para fazer uma atividade e as variáveis $31 \mathrm{~b}$ e $31 \mathrm{c}$ referem-se a quantidade de acessos do aluno ao ambiente.

O último modelo refere-se ao construto Estabelecimento de Metas (EM). Para este construto, das três variáveis comportamentais escolhidas, duas delas (VAR33 e $V A R 44)$ tem significância estatística para representar o construto estabelecimento de metas. A variável (VAR33) refere-se a quantidade de atividades entregues por um(a) aluno(a) no prazo. A variável 34 refere-se a Quantidade geral de postagens de um(a) aluno(a) em fóruns. De acordo com a análise, podemos afirmar que ambas as atividades tem significância estatística para representar o construto Estabelecimento de metas.

Ao final da aplicação dos seis modelos de regressão obtivemos um total de treze variáveis significativas referente ao comportamento de autorregulação da aprendizagem dos alunos na plataforma Moodle.

\section{Considerações Finais}

Ainda é pouco expressiva a quantidade de pesquisas sobre a mensuração das características de autorregulação da aprendizagem com foco em ambientes de EAD. A maioria dos instrumentos de coleta disponibilizados na literatura para a mensuração de SRL foram desenvolvidos para ambientes presenciais de ensino.

Os resultados desta pesquisa vem a contribuir com a comunidade acadêmica de Mineração de Dados Educacionais bem como a comunidade de pesquisadores que buscam entender os fenômenos de autorregulação da aprendizagem, especificamente em contextos da aprendizagem à distância.

A validação das variáveis comportamentais, possibilitará que outros pesquisadores possam aplicar métodos da estatística e mineração de dados para a construção de modelos preditivos capazes de dar indícios de previsibilidade ao professor para tomada de decisão e condução da ação docente, sobre o comportamento de autorregulação da aprendizagem dos alunos.

\section{Agradecimentos}

Esta pesquisa é apoiada pela FACEPE-APQ No. 0525-1.03/14. Os autores também agradecem ao apoio do NEAD/UPE, PRPPG/UFRPE (006999/2015-28) e PRPPGI/UNIVASF (Edital 18/2013). Alex Sandro Gomes é bolsista DT Nível 2/CNPq, processos no $310466 / 2012-1$ e n ${ }^{\circ} 475634 / 2013-6$.

\section{Referências}

Adesope, O O, M Zhou, e J C Nesbit. "Achievement Goal Orientations and Self-Reported Study Strategies as Predictors of Online Studying Activities." Journal of Educational Computing Research, 2015: 436-458.

Ali, L, M Hatala, D Gašević, e P H Winne. "Leveraging MSLQ Data for Predicting Students Achievement Goal Orientations." Journal of Learning Analytics, 2014: 157-160.

Azevedo, R. "Using hypermedia as a metacognitive tool for enhancing student learning? The role of self-regulated learning." Educational Psychologist, 2005: 199-209. 
V Congresso Brasileiro de Informática na Educação (CBIE 2016)

Anais do XXVII Simpósio Brasileiro de Informática na Educação (SBIE 2016)

Barnard, L, W Y Lan, S M Crooks, e V O Paton, V. O. The relationship of epistemological beliefs with self-regulatory skills in the online course environment. Journal of Online and Learning Teaching, 2008.

Barnard, L, W Y Lan, Y M To, e V O Paton. "Measuring self-regulation in online and blended learning environments." The Internet and Higher Education, 2009: 1-6.

Barnard, L, W Y Lan, Y M To, V O Paton, e S L Lai. Measuring self-regulation in online and blended learning environments. The Internet and Higher Education, 2009.

Bolfarine, H, e O H Bussab. Elementos de amostragem. São Paulo: Edgard Blücher, 2005.

Bondareva, D, C Conati, R Feyzi-Behnagh, J M Harley, R Azevedo, e F Bouchet. "Inferring learning from gaze data during interaction with an environment to support selfregulated learning." In Artificial Intelligence in Education , 2013: 229-238.

Broadbent, J, e W L Poon. "Self-regulated learning strategies \& academic achievement in online higher education learning environments: A systematic review." The Internet and Higher Education, $\mathrm{n}^{\mathrm{o}} 27$ (2015): 1-13.

Cameron, A C, e P K Trivedi. Regression analysis of count data. Cambridge University Press, 1998.

Chen, C. S. Self-regulated learning strategies and achievement in an introduction to information systems course. Information Technology, Learning and Performance Journal, 2002.

Dabbagh, N, e A Kitsantas. "Supporting self-regulation in student-centered web-based learning environments." International Journal on E-learning, 2004: 40-47.

Dabbagh, N, e A Kitsantas. "Using web-based pedagogical tools as scaffolds for selfregulated learning." Instructional Science, 2005: 513-540.

Freitas, A. L P, e S G Rodrigues. A avaliação da confiabilidade de questionário: uma análise utilizando o coeficiente alfa de Cronbach. XII SIMPEP, 2005.

Mato, C A, e R T Veiga. Avaliação da qualidade percebida de serviços: um estudo em uma organização não-governamental. São Paulo: Caderno de Pesquisa em Administração, 2000.

Miguel, P. A C, e G E Salomi. Uma revisão dos modelos de medição de qualidade em serviços. Rio de Janeiro: Revista Produção, 2004.

Milan, G S, e G Trez. Pesquisa de satisfação: um modelo para planos de saúde. RAE Eletrônica, Revista de Administração de Empresas, 2005.

Nussbaumer, A, E C Hillemann, C Gütl, e D Albert. "A competence-based service for supporting self-regulated learning in virtual environments." Journal of Learning Analytics, 2015: 101-133.

Oviedo, H C, e A Campo-Arias. Aproximación al uso del coeficiente alfa de Cronbach. Revista Colombiana de Psiquiatría, 2005.

Panadero, E, P A Kirschner, S Järvelä, J Malmberg, e H Järvenoja. "How Individual SelfRegulation Affects Group Regulation and Performance A Shared Regulation Intervention." Small Group Research, 2015. 
V Congresso Brasileiro de Informática na Educação (CBIE 2016)

Anais do XXVII Simpósio Brasileiro de Informática na Educação (SBIE 2016)

Pintrich, P. R. The role of motivation in promoting and sustaining self-regulated learning. International Journal of Educational Research, 1999.

Sabourin, J L, B W Mott, e J C Lester. "Early Prediction of Student Self-Regulation Strategies by Combining Multiple Models." International Educational Data Mining Society, 2012.

Salomi, G E, P. A C Miguel, e A J Abackerli. SERVQUAL x SERVPERF: comparação entre instrumentos para avaliação da qualidade de serviços internos. Gestão \& Produção, 2005.

Sanchez-Santillan, M, M Paule-Ruiz, R Cerezo, e V Alvarez-García. "MeL: Modelo de adaptación dinámica del proceso de aprendizaje en eLearning." anales de psicología, 2016: 106-114.

Schoor, C, e M Bannert. "Exploring regulatory processes during a computer-supported collaborative learning task using process mining." Computers in Human Behavior, 2012: 1321-1331.

Schunk, D H. Self-regulated learning The educational legacy of Paul R. Pintrich. Educational Psychologist, 2005.

Schunk, D H, e B J Zimmerman. "Self-regulated learning: From teaching to self-reflective practice." Guilford Press, 1998.

Segedy, J R, J S Kinnebrew, e G Biswas. "Using coherence analysis to characterize selfregulated learning behaviours in open-ended learning environments." 2015: 13-48.

Sonnenberg, C, e M Bannert. "Discovering the Effects of Metacognitive Prompts on the Sequential Structure of SRL-Processes Using Process Mining Techniques.” Journal of Learning Analytics, 2015: 72-100.

Urdan, A T. Qualidade de Serviços médicos na perspectiva do cliente. Revista de Administração de Empresas, 2001.

Winne, P H, e D Jamieson-Noel. "Exploring students' calibration of self reports about study tactics and achievement." Contemporary Educational Psychology, 2002: 551-572.

Winne, P H, e N E Perry. "Measuring self-regulated learning." Handbook of self-regulation, 2000: 531-566.

You, J. W. "Identifying significant indicators using LMS data to predict course achievement in online learning." The Internet and Higher Education, 2016: 23-30.

Yukselturk, E, e S Bulut. "Gender differences in self-regulated online learning environment." Journal of Educational Technology \& Society, 2009: 12-22.

Zimmerman, B J, e M Martinez-Pons. Construct validation of a strategy model of student self-regulated learning. Journal of Educational Psychology, 1988. 